\title{
Trend of bacterial meningitis in Bahrain from 1990 to 2013 and effect of introduction of new vaccines
}

\author{
N. Saeed, ${ }^{1}$ H. AlAnsari, ${ }^{2}$ S. AlKhawaja,, 3 J.S. Jawad, ${ }^{4}$ K. Nasser ${ }^{3}$ and E. AlYousef ${ }^{5}$
}

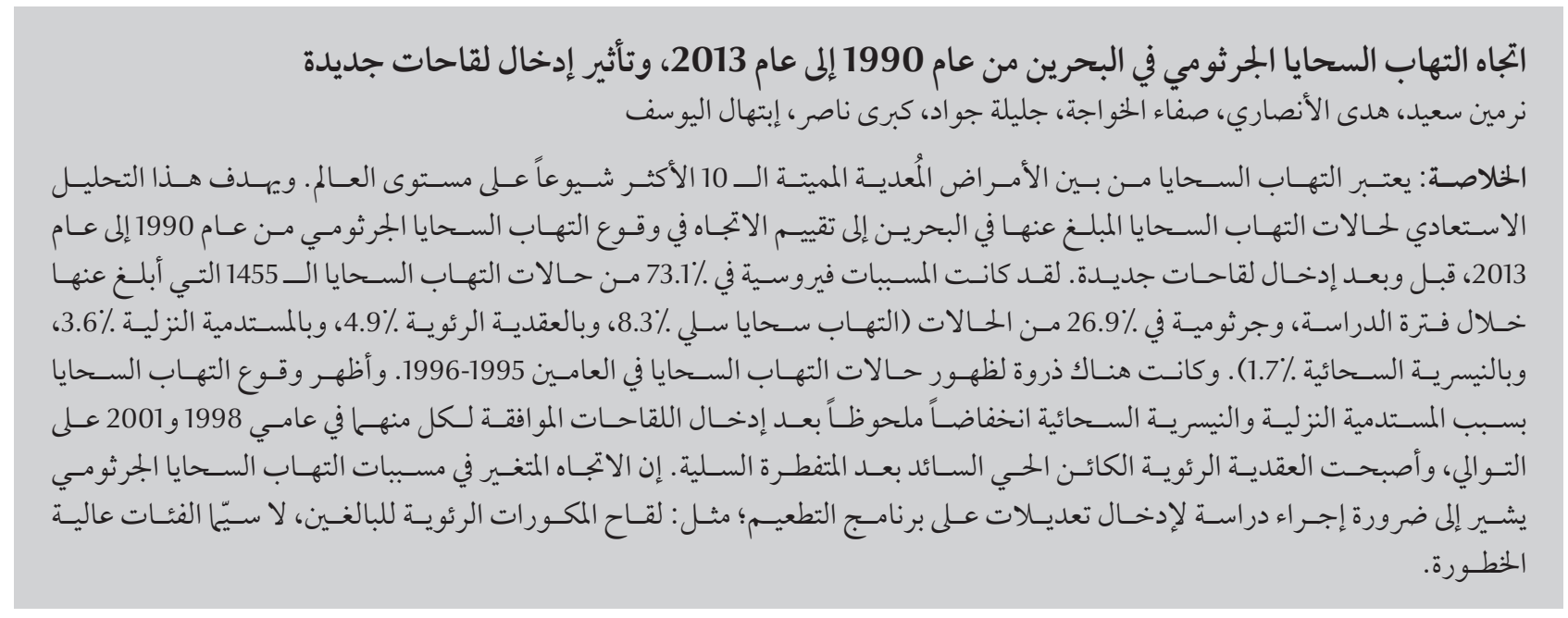

ABSTRACT Meningitis is among the 10 commonest infectious causes of death worldwide. This retrospective analysis of reported cases of meningitis in Bahrain aimed to assess the trend in the incidence of bacterial meningitis from 1990 to 2013, before and after the introduction of new vaccines. Of 1455 reported cases of meningitis during the study period $73.1 \%$ were viral and $26.9 \%$ were bacterial etiology (tuberculous meningitis 8.3\%; Streptococcus pneumoniae 4.9\%, Haemophilus influenzae 3.6\% and Neisseria meningitidis 1.7\%). There was a peak of meningitis cases in 1995-1996. The incidence of meningitis due to $H$. influenzae and $N$. meningitidis showed a marked reduction after the introduction of the corresponding vaccines in 1998 and 2001 respectively, and S. pneumoniae became the predominant organism after Mycobacterium tuberculosis. The changing trend in the etiology of bacterial meningitis points to the need to study vaccination programme modifications, such as pneumococcal vaccine for the adult population, especially high-risk groups.

Tendances de la méningite bactérienne à Bahreïn entre 1990 et 2013 et effets de l'introduction de nouveaux vaccins

RÉSUMÉ La méningite fait partie des 10 causes de décès par infection les plus courantes dans le monde. La présente analyse rétrospective des cas de méningite notifiés à Bahreïn visait à mesurer la tendance de l'incidence de la méningite bactérienne entre 1990 et 2013, avant et après l'introduction de nouveaux vaccins. Sur 1455 cas de méningite rapportés au cours de la période d'étude, 73,1\% étaient d'étiologie virale et 26,9\% d'étiologie bactérienne (méningite tuberculeuse 8,3\% ; Streptococcus pneumoniae 4,9\%, Haemophilus influenzae 3,6\% et Neisseria meningitidis 1,7 \%). Un pic de cas de méningite est survenu entre 1995 et 1996. L'incidence des méningites dues à $H$. influenzae et $N$. meningitidis a marqué une nette réduction après l'introduction des vaccins correspondants en 1998 et 2001 respectivement, et $S$. pneumoniae est devenu l'organisme prédominant après Mycobacterium tuberculosis. Ce changement de tendance dans l'étiologie de la méningite bactérienne souligne le besoin d'étudier les possibilités de modifications du programme de vaccination, telle que la vaccination antipneumococcique de la population adulte, notamment pour les groupes à haut risque.

${ }^{7}$ Department of Pathology; ${ }^{2}$ Department of Infection Control; ${ }^{3}$ Communicable Disease Section; ${ }^{4}$ Immunization Section, Ministry of Health, Bahrain. ${ }^{5}$ Department of Internal Medicine, Salmaniya Medical Centre, Bahrain (Correspondence to N. Saeed: nkamalh@hotmail.com). Received: 25/08/15; accepted: 10/01/16 


\section{Introduction}

Despite improvements in its treatment, bacterial meningitis continues to have high mortality and morbidity rates worldwide. Meningitis is among the 10 most common infectious causes of death and is responsible for approximately 135000 deaths annually throughout the world. Significant neurological sequelae are frequently encountered among the survivors (1). Streptococcus pneumoniae and Neisseria meningitidis are the main pathogens of community-acquired bacterial meningitis in adults. However, the epidemiology of bacterial meningitis continues to shift with the ongoing introduction of vaccines against the most common pathogens of bacterial meningitis (2).

Bahrain is an island on the east coast of the Arabian Peninsula with a population of 1.31 million in the year 2013. Immigrants make up about $55 \%$ of the total population (3). Bahrain has one of the most efficient health care systems in the World Health Organization (WHO) Eastern Mediterranean Region. Bahrain implements a comprehensive expanded immunization programme with excellent coverage rates (> 95\%) for most routine childhood vaccines, including those against $S$. pneumoniae, N. meningitidis and Haemophilus influenzae. $H$. influenzae vaccine was introduced in routine childhood vaccines in 1998, and bivalent meningococcal AC vaccine was introduced in 1995 for children aged 2 years and for those travelling for pilgrimage. However, it was replaced in 2000 by the quadrivalent meningococcal ACYW vaccine. One year later, in 2001, the conjugated pneumococcal vaccine was introduced for high-risk people and was incorporated into the routine childhood vaccination schedule by 2008 (4).

Epidemiological surveys are important to document the incidence of bacterial meningitis in the periods before and after the introduction of vaccines to a country. This could allow proper evaluation of the impact of new vaccines by considering the geographical variations in incidence of the causative pathogens across countries. It could also help to detect a need for modification of the vaccination protocol if the efficacy of the current protocol is inadequate (5). According to the best of our knowledge, there have been no previous surveillance studies to monitor the efficacy of the current vaccination protocols applied in Bahrain to prevent bacterial meningitis. This stimulated us to conduct this study to assess the trend and the temporal relationship between the introduction of new vaccines and the change in the incidence of bacterial meningitis over 24 year-period 1990 to 2013.

\section{Methods}

\section{Study design and data source}

This study was a retrospective analysis of reported cases of bacterial meningitis in Bahrain from 1990 to 2013. Data were retrieved from the national surveillance system of communicable diseases, which is managed by the Department of Public Health at the Ministry of Health of Bahrain. The meningitis surveillance includes both passive and active surveillance systems. The research and ethics committee of Salmaniya Medical Complex approved the study. This was a purely record-based study with no ethical issues and no consent was taken.

\section{Data collection}

Passive surveillance includes mandatory reporting for all the clinically suspected and confirmed meningitis cases reported from all government and private hospitals in the country. All the microbiology laboratories in Bahrain also report any positive cerebrospinal fluid (CSF) culture from laboratory confirmed cases. Clinically suspected cases are defined as illness with sudden onset of fever $\left(>38.5^{\circ} \mathrm{C}\right)$ and one of the following (neck stiffness, altered consciousness, signs of meningeal irritation or petechial/purpuric rash) or bulging fontanel in infants. Cases are then stratified into either probable (when combined with turbid CSF) or confirmed (when accompanied by positive CSF culture) according to WHO case definitions and laboratory criteria (6). Active surveillance for meningitis cases was done through regular tracing and review of medical records based on International Classification of Diseases coding for inpatients admitted to Salmaniya Medical Complex, which is the only government tertiary care hospital concerned with treatment of such cases in the country. In the current study, we included only the confirmed cases. Meningitis cases were also classified by etiology into viral or bacterial and type of bacterium.

\section{Data analysis}

Data pertaining to all cases were entered into a Microsoft Excel database. The data were analysed separately for the trend of change in the incidence over the last 24 years using TexaSoft WINKS SDA software 2007 (6th edition). Comparisons between the number of meningitis cases in the periods before and after the introduction of specific vaccines into the Bahrain vaccination programme were performed with Student $t$-test, with $P<$ 0.05 considered statistically significant.

\section{Results}

\section{All types of meningitis}

Table 1 shows the total number of cases of meningitis reported in Bahrain from 1990 to 2013 and the number of cases by etiology. The total number of meningitis cases during the study period was 1437. Viral meningitis accounted for $1051(73.1 \%)$ cases and bacterial meningitis for 386 (26.9\%) cases. Tuberculous meningitis was the most frequent bacterial cause, detected in 122 patients ( $8.5 \%)$, followed by S. pneumoniae (70 cases; 4.9\%), H. influenzae (52 


\begin{tabular}{|c|c|c|c|c|c|c|c|c|c|}
\hline \multirow[t]{2}{*}{ Year } & \multirow[t]{2}{*}{ Total } & \multirow[t]{2}{*}{ Viral } & \multirow[t]{2}{*}{ Bacterial } & \multicolumn{6}{|c|}{ Subtype of bacterial meningitis } \\
\hline & & & & Pneumococcal & Meningococcal & $\begin{array}{l}H . \text { influenzae } \\
\text { type b }\end{array}$ & Staphylococcal & Tuberculous & Other \\
\hline 1990 & 22 & 7 & 15 & 4 & 4 & 2 & 0 & 5 & 0 \\
\hline 1991 & 45 & 32 & 13 & 4 & 0 & 4 & 1 & 1 & 3 \\
\hline 1992 & 43 & 14 & 29 & 6 & 4 & 5 & 1 & 4 & 9 \\
\hline 1993 & 26 & 10 & 16 & 2 & 1 & 3 & 0 & 4 & 6 \\
\hline 1994 & 31 & 20 & 11 & 2 & 2 & 2 & 0 & 4 & 1 \\
\hline 1995 & 310 & 285 & 25 & 2 & 0 & 9 & 0 & 10 & 4 \\
\hline 1996 & 232 & 199 & 33 & 1 & 1 & 11 & 0 & 8 & 12 \\
\hline 1997 & 44 & 25 & 19 & 1 & 2 & 7 & 0 & 2 & 7 \\
\hline 1998 & 46 & 27 & 19 & 5 & 4 & 4 & 0 & 5 & 1 \\
\hline 1999 & 55 & 40 & 15 & 3 & 1 & 2 & 0 & 6 & 3 \\
\hline 2000 & 68 & 54 & 14 & 2 & 1 & 3 & 1 & 6 & 1 \\
\hline 2001 & 45 & 35 & 10 & 2 & 3 & 0 & 0 & 1 & 4 \\
\hline 2002 & 40 & 32 & 8 & 4 & 0 & 0 & 0 & 2 & 2 \\
\hline 2003 & 46 & 38 & 8 & 5 & 0 & 0 & 0 & 3 & 0 \\
\hline 2004 & 40 & 26 & 14 & 3 & 0 & 0 & 1 & 10 & 0 \\
\hline 2005 & 22 & 12 & 10 & 2 & 0 & 0 & 3 & 2 & 3 \\
\hline 2006 & 57 & 39 & 18 & 4 & 0 & 0 & 0 & 7 & 7 \\
\hline 2007 & 38 & 27 & 11 & 2 & 0 & 0 & 1 & 6 & 2 \\
\hline 2008 & 33 & 15 & 18 & 4 & 0 & 0 & 0 & 6 & 8 \\
\hline 2009 & 47 & 27 & 20 & 1 & 0 & 0 & 0 & 13 & 6 \\
\hline 2010 & 37 & 23 & 14 & 3 & 1 & 0 & 2 & 5 & 3 \\
\hline 2011 & 10 & 0 & 10 & 3 & 0 & 0 & 0 & 3 & 4 \\
\hline 2012 & 37 & 18 & 19 & 4 & 0 & 0 & 1 & 7 & 7 \\
\hline 2013 & 63 & 46 & 17 & 1 & 0 & 0 & 1 & 2 & 13 \\
\hline Total & 1437 & 1051 & 386 & 70 & 24 & 52 & 12 & 122 & 106 \\
\hline
\end{tabular}

cases; 3.6\%) and then N. meningitidis (24 cases; $1.7 \%$ ).

Figure 1 illustrates the trend of the viral and bacterial meningitis during the study period. The average incidence of meningitis between 1990 and 2000 ranged from around 5 to 10 cases per 100000 population (Figure 1). The trend was almost stable for viral and bacterial meningitis during the study period. However, the incidence showed a marked peak in 1995 and 1996, reaching 50 cases per 100000 population, mostly due to viral and $H$. influenzae meningitis. From 2001, there was gradual decline in the incidence of meningitis from 6.7 cases per 100000 in 2001 to 5.0 cases per 100000 in 2013 (Figure 1).

\section{Bacterial meningitis}

Figure 2 shows the incidence of pneumococcal, meningococcal and Haemophilus influenzae meningitis in both adults and children over the 24year period. Figure 3 shows the trend in the incidence of tuberculous and total bacterial meningitis and of pneumococcal, meningococcal and Haemophilus influenzae meningitis in children only. The charts illustrate the decline in the incidence of different types of bacterial meningitis after the introduction of the corresponding vaccines.

\section{Pneumococcal meningitis}

Figure 2 shows that the incidence of pneumococcal meningitis was 1 per 100000 population at the beginning of study period in 1990 and dropped gradually to 0.1 per 100000 in 2013. Figure 3 shows the trends in incidence in children only. The red arrows refer to the timing of various stages of introduction of pneumococcal conjugate vaccine. The first arrow (2001) refers to the introduction of the vaccine to high-risk children, while the second red arrow (2008) indicates the expansion of the 7 valent pneumococcal conjugate vaccine to include all infants through routine childhood vaccination (at ages 2, 4, 6 and 18 months). The third red arrow (2010) refers to replacing the 7 polyvalent vaccines with 13 polyvalent vaccines for children. The fourth red arrow (2013) refers to the introduction of 13 polyvalent vaccines for adults aged $\geq 50$ years. 


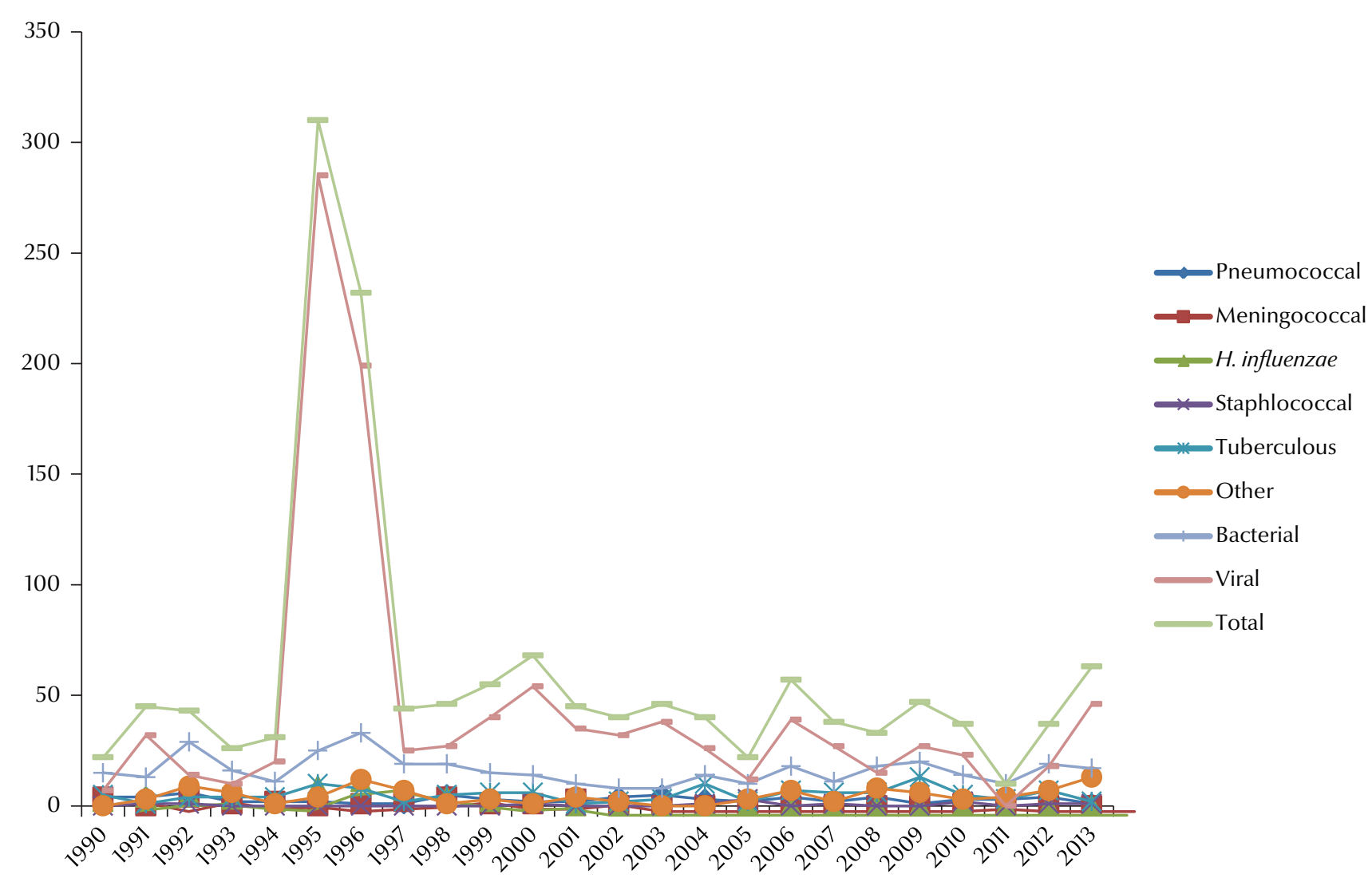

Figure 1 Annual incidence of total, viral and bacterial meningitis per 100000 population in Bahrain over the years 1990-2013

Figure 4 shows the percentage of paediatric versus adult cases of pneumococcal meningitis. There was significant reduction of the number of reported cases of pneumococcal meningitis from 58 cases before the introduction of pneumococcal vaccine in 2008 to only 12 cases from 2009 to 2013 after introduction of the vaccine (Table 1). Four cases occurred in children $(<14$ years old); all of them were infants below the age of 1 year, and 2 of them were unvaccinated 5-day-old neonates with positive blood and CSF culture. Three cases were 7,8 and 11 months old; 2 of them had received the recommended pneumococcal vaccine on time (at 2, 4 and 6 months of age). The third case was an immigrant non-Bahraini infant who had not previously received pneumococcal vaccination as this vaccine was not included in the routine vaccination schedule in her original country. It is also worth mentioning that all the cases of pneumococcal meningitis recorded before 2008 were in children. However, the proportion of paediatric cases with pneumococcal meningitis gradually decreased to $62 \%$ after introduction of the pneumococcal vaccine.

\section{Haemophilus meningitis}

A total of 52 cases of $H$. influenzae meningitis were reported during the 24-year period of the study (Table 1). Most cases of $H$. influenzae meningitis (44 cases) were reported before the introduction of $H$. influenzae vaccine for childhood in 1998. In 1995, there were 9 cases with $H$. influenzae meningitis and 3 cases with other unknown bacterial causes, while in 199611 cases with H. influenzae meningitis were reported among children aged $0-4$ years ( 15 per 100000$)$ and 9 cases with other unknown bacterial causes with negative culture and no identification.

Figure 2 shows that the incidence of $H$. influenzae meningitis was 0.4 cases per 100000 population in 1990, which reached a peak of 1.9 cases per 100000 in 1996 with a cluster of cases and a possible outbreak.

With introduction of the $H$. influenzae conjugate vaccine to routine childhood vaccination in 1998 (shown by the green arrow on the chart) there was a marked reduction of the incidence of reported cases to reach zero after 2001 where there was no single reported case of H. influenzae meningitis in Bahrain.

\section{Meningococcal meningitis}

There were 24 cases of meningococcal meningitis reported during the study period (Table 1). Figure 2 shows that the incidence of meningococcal meningitis was 0.83 per 100000 population in 1990 and that this fluctuated annually until 2001.

Figure 3 showed the trend of meningococcal meningitis in children and the related decline in its incidence related to the introduction of the corresponding vaccine. The first blue arrow (1995) 


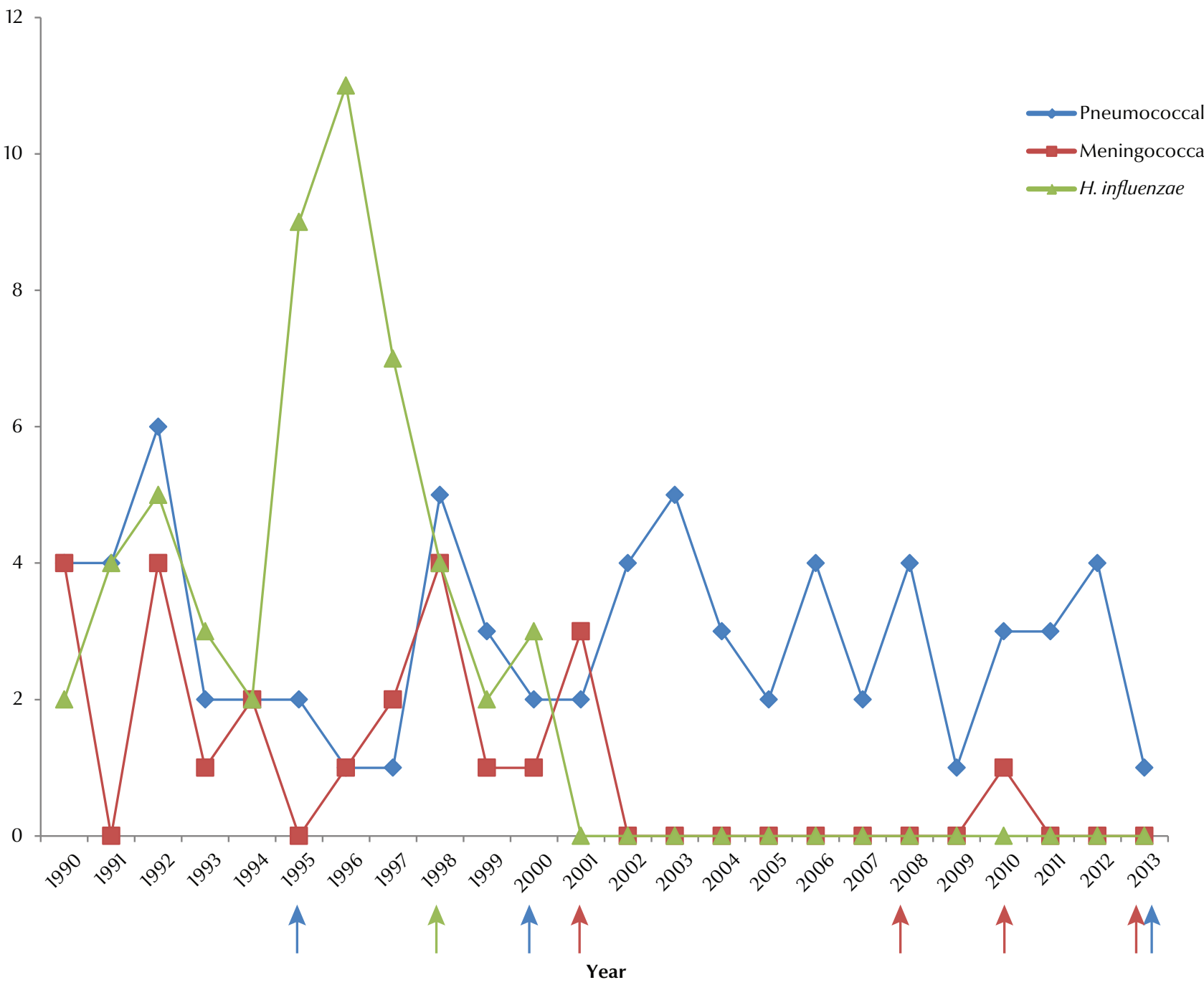

Figure 2 Annual incidence of pneumococcal, meningococcal and Haemophilus influenzae bacterial meningitis per 100000 population in Bahrain over the years 1990-2013. Arrows indicate dates of introduction of specific vaccines into the national programme of immunization (red arrows = various stages of introduction of pneumococcal conjugate vaccine; green arrow = introduction of $\boldsymbol{H}$. influenza conjugate vaccine; blue arrows = various stages of introduction of meningococcal vaccine)

refers to the timing of introduction of meningococcal AC vaccine routinely for children at age 2 years and for hajj pilgrims, while the second blue arrow (2000) refers to the replacement of meningococcal AC by meningococcal ACWY polysaccharide for the same target group, and the third blue arrow (2013) indicates the change from quadrivalent meningococcal polysaccharide to the meningococcal ACWY conjugate for children aged 2 years, hajj pilgrims and high-risk groups. There were no reported cases of meningococcal meningitis after 2002 (1 year after the introduction of meningococcal
ACWY), except in 2010 when there 1 case of meningococcal meningitis reported in a 44-year-old Bahraini male. Although the patient had no known risk factor or travel history, he was working at the airport and we cannot exclude the possibility of exposure to an infected traveller.

\section{Discussion}

The study aimed to investigate the annual trends and the effect of vaccination on the incidence of bacterial meningitis in Bahrain. It showed a gradual decline in the overall annual incidence of meningitis (bacterial and viral) during the study period from 1990 to 2013 . The overall annual incidence ranged from 5 to 10 per 100000 population, which is close to the incidence in nearby Gulf Cooperation Council (GCC) countries such as Qatar (10 per 100 000) (7) and Oman (3 to 8.4 per 100000$)(8)$, but much higher than observed in United Arab Emirates (1 to 2.2 per 100000 ) (9).

We noted a peak number of reported confirmed cases with meningitis during 1995 and 1996, mostly due to viral and $H$. influenzae meningitis. This outbreak was the stimulus to introduce 


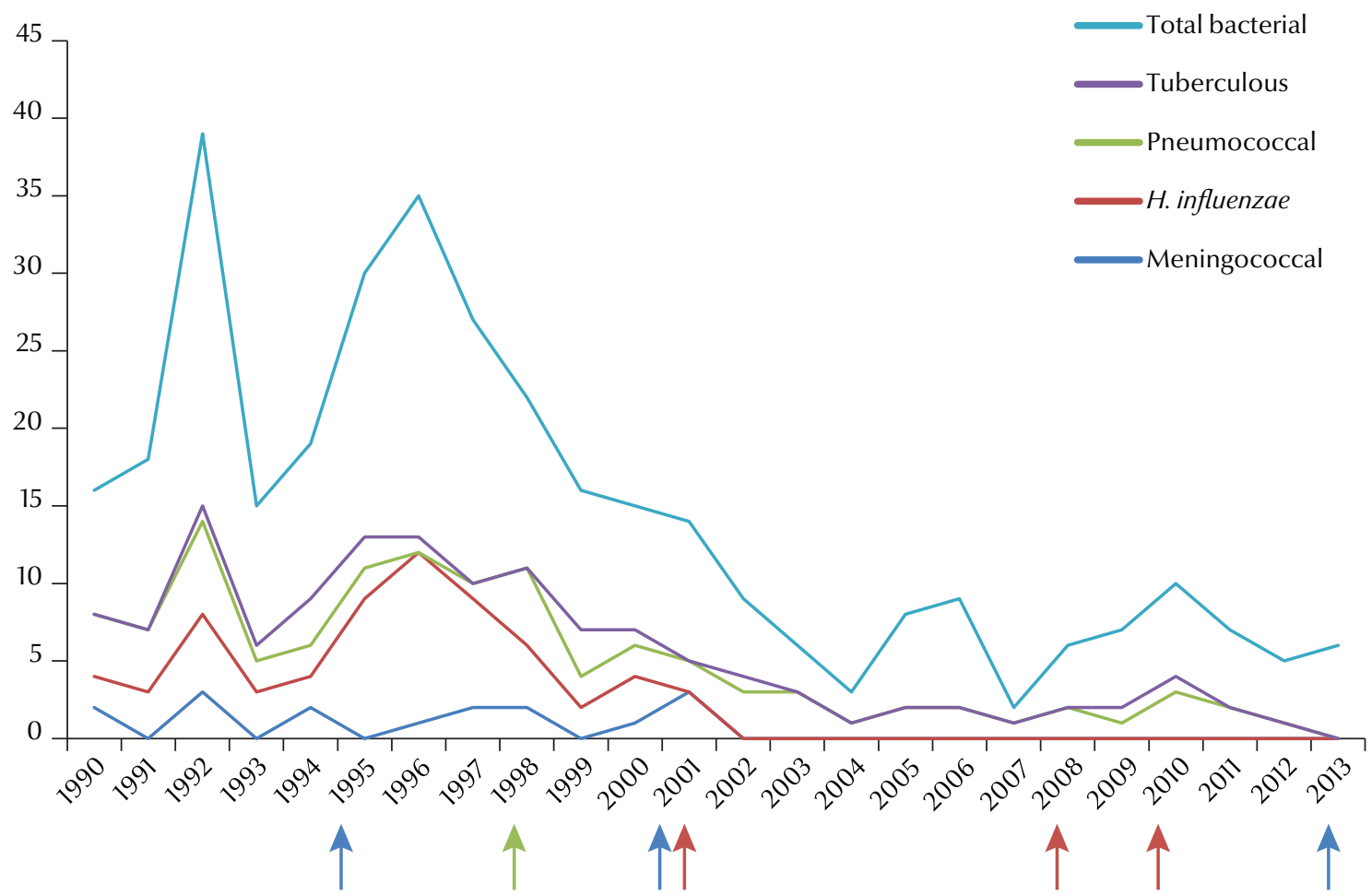

Figure 3 Annual incidence of pneumococcal, meningococcal, Haemophilus influenzae, tuberculous and total bacterial meningitis per 100000 children in Bahrain over the years 1990-2013. Arrows indicate dates of introduction of specific vaccines into the national programme of immunization (red arrows = various stages of introduction of pneumococcal conjugate vaccine; green arrow = introduction of $H$. influenzae conjugate vaccine; blue arrows = various stages of introduction of meningococcal vaccine)

the H. influenzae type b (Hib) vaccine in 1998 as a part of the 5-antigen vaccine (against diphtheria, tetanus, pertussis, hepatitis $B$ and $H$. influenzae type $b$ ) (10).

The epidemiology of bacterial meningitis in Bahrain continues to change with the implementation of vaccination strategies that target the most common community-acquired pathogens. During the study period (1990-2013), the most pivotal changes were observed with the introduction of $H$. influenzae vaccines in 1998 and N. meningitidis vaccine in 2000. Introduction of these two vaccines has been associated with a striking reduction in the incidence of both $H$. influenzae and N. meningitidis, and since the year 2000 there was no single reported case of $H$. influenzae and since 2002 onward there was only 1 reported case of $N$. meningitidis meningitis. This temporal relationship and the marked reduction in the rate of meningitis due to $H$. influenzae early after the introduction of vaccine has been also observed in other nearby countries from the GCC region as well other developed counties (11). For example, in Kuwait, H. influenzae was responsible for nearly half of the meningitis cases at the early 1990s, prior to the introduction of routine $\mathrm{Hib}$ vaccination. However, it was replaced by S. pneumoniae as the leading causative agent for bacterial meningitis in Kuwait after implementation of the successful Hib vaccination policy (12). The same observation was also noted worldwide. In the United States of America there was a significant decline in the incidence of H. influenzae meningitis over all States from 0.10 per 100000 population in 1997 to 0.058 per 100000 in 2010 (1315). England showed also significant reduction in hospital admission rates for childhood invasive bacterial disease including meningitis after the introduction of conjugate vaccines against
H. influenzae, N. meningitidis and S. pneumoniae (16).

N. meningitidis is responsible for serious cases of meningitis and fulminant septicaemia and is also a concern due to the characteristic dynamic nature of the disease, both in its tendency to occur in outbreaks and epidemics as well as the fact that the most prominent disease-causing serogroups can change in a given region over a period of only few years (17). The current study also revealed a great reduction in the incidence of meningococcal meningitis in Bahrain from 0.83 per 100000 in 1990 followed by a fluctuating pattern until 2001, which then reached zero incidence after 2002, 1 year after the introduction of meningococcal ACWY polysaccharide for children. However, 1 case of meningococcal meningitis was reported in a 44-year-old healthy male adult in 2010 with no history of travel or contact with sick patients with a similar 


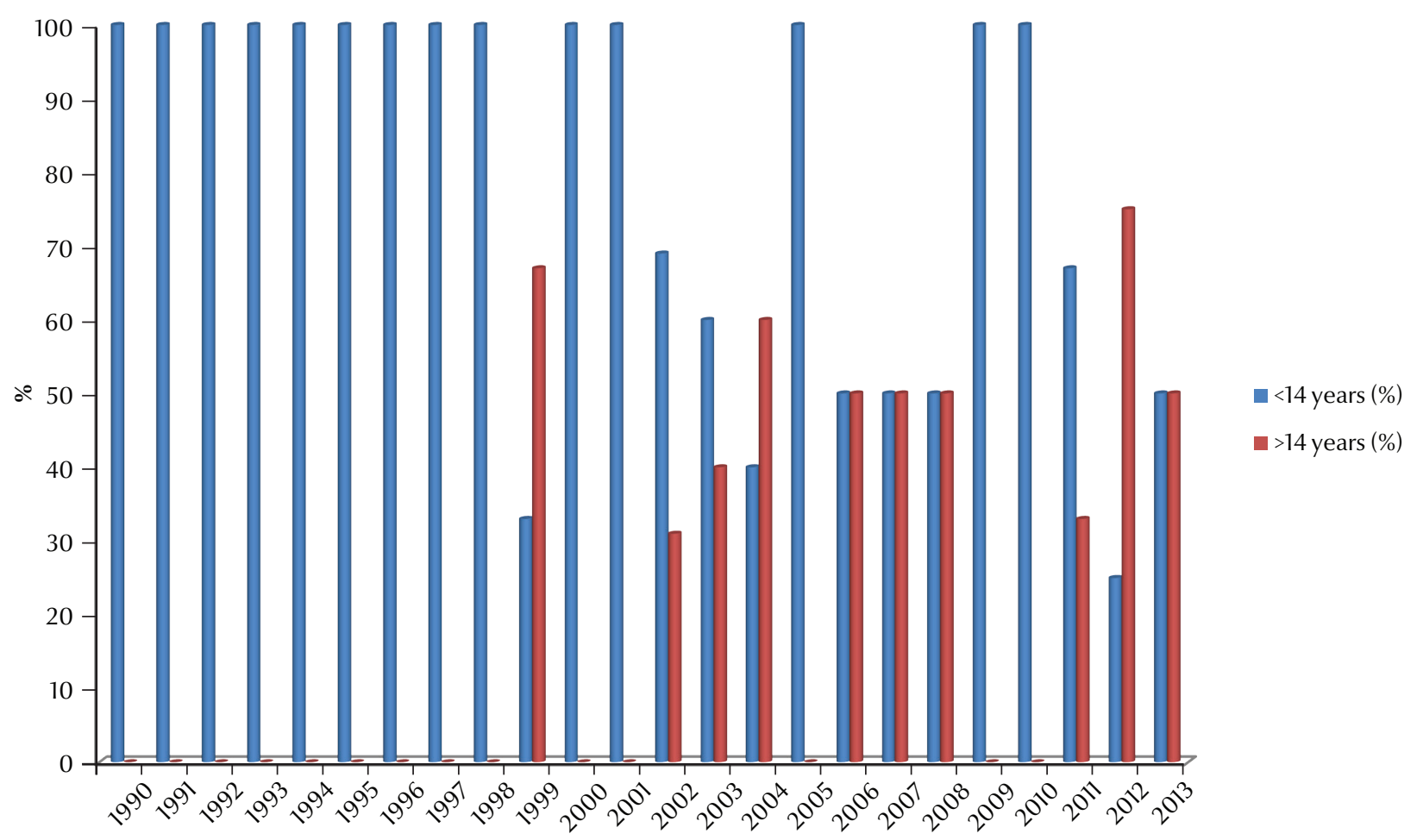

Figure 4 Percentage of paediatric versus adult cases of pneumococcal meningitis in Bahrain over the years 1990-2013

illness. Such a major reduction in the incidence of meningococcal meningitis after the introduction of meningococcal ACWY vaccine and without changes in the meningitis control programme in our country is an indicator of the adequacy and efficiency of the country's vaccination policy. Similar data in other GCC countries showed a reduction of meningococcal disease but to a lesser degree than observed in the current study in Bahrain. Bahrain has a small population which allows adequate implementation of the vaccination programme, with a direct impact of the vaccine on the target population as well as an indirect protection of others due to a decrease in the risk of disease contact, reduced transmission rate and increase herd immunity in the community. We noted that Oman and Qatar all reported incidence rates below 2 per 100000 , with the exception of a few higher rates in the mid-1980s (18). However, we cannot compare our results with those from Qatar as their study included only children aged $<12$ years. Other countries outside the Gulf region such as the USA also reported the efficacy of the meningococcal vaccine, with a great impact of the vaccine on reduction of the incidence of meningococcal disease (19).

Our study also illustrated a significant drop in the incidence of S. pneumoniae meningitis in Bahrain from 1 per 100000 population at the beginning of study period in 1990 to 0.1 per 100000 in 2013. Yet it became the leading cause of bacterial meningitis (not considering tuberculous meningitis) in Bahrain, replacing $H$. influenzae and $N$. meningitidis after the striking reduction of these two organisms as a cause of bacterial meningitis following the introduction of the corresponding vaccines. The age distribution of S. pneumoniae meningitis showed significant differences between 1990 and 2013. The disease was more frequent in the paediatric age group (<14 years of age) at the beginning of the study; but after integrating the $S$ pneumoniae vaccine into the extended immunization programme for children the disease started to be less common among the paediatric age group, and adults became the main target. This shift may indicate a need to improve the vaccination strategy, especially for highrisk adults in Bahrain, given that many Bahrainis have sickle-cell disease which poses a significant risk of invasive pneumococcal disease. Our finding agrees with the work of Castelblanc et al., who observed a similar reduction in the incidence of $S$. pneumoniae meningitis and a change in its epidemiological pattern with a reduction of incidence from 0.81 per 100000 in 1997 to 0.3 per 100000 in 2010. However, they showed a consistent reduction in incidence in all age groups, with a greater reduction in children younger than 18 years of age (20). The same finding was also noted in a similar study in South Africa, which showed a substantial decrease in the incidence of invasive pneumococcal disease, particularly among children, after the introduction of pneumococcal vaccine into the routine childhood schedule (21). The expansion of the vaccine to adults and elderly people was 
recommended and supported by other worldwide studies for further reduction of the rate of invasive pneumococcal disease (22-25).

The limitation of our study was that we did not know the serotypes of pneumococcal isolates causing pneumococcal meningitis after incorporation of pneumococcal vaccine into the routine childhood schedule. This would have enabled us to capture a decrease or near disappearance of vaccine serotypes or the emergence of non-vaccine serotypes and would have informed possible modifications to our vaccine policy.

\section{Conclusion}

The significant reduction of invasive $H$. influenzae meningitis since 2000, and the parallel resurgence of $S$. pneumoniae as the main cause of bacterial meningitis with an increased proportion of adult cases, provide evidence of the efficacy of the childhood vaccination programme in Bahrain against both $H$. influenzae and N. meningitidis. At the same time, it stimulates us to study the possibility of modification to the vaccination programme, such as including pneumococcal vaccine for the adult population, especially for high-risk groups, to prevent invasive pneumococcal disease. Funding: None.

Competing interests: None declared.

\section{References}

1. Scheld WM, Koedel U, Nathan B, Pfister HW. Pathophysiology of bacterial meningitis: mechanism(s) of neuronal injury. J Infect Dis. 2002 Dec 1;186 Suppl 2:S225-33. PMID:12424702

2. Shah SF, Nadeem S, Farooq A, Bener A. Emerging trend in the epidemiology of meningitis in Qatar. J Coll Physicians Surg Pak. 2008 Apr;18(4):259-60. PMID:18474169

3. Population and demographics [Internet]. Manama, Bahrain: Ministry of Information Affairs (www.mia.gov.bh/en/Kingdomof-Bahrain/Pages/Population-and-Demographic-Growth. aspx, accessed 19 January 2016).

4. Six countries have decided to routinely immunise children against pneumococcal disease with Prevenar. PharmacoEconomics \& Outcomes News. 2008 Jul;558(1):4.

5. Travasso C. Better surveillance and vaccine coverage are needed to eliminate measles in India by 2020. BMJ. 2015;350:h900. PMID:25701024

6. WHO-recommended standards for surveillance of selected vaccine-preventable diseases. Geneva: World Health Organization; 2003. (WHO/V\&B/03.01) (http://www.measlesrubellainitiative.org/wp-content/uploads/2013/06/ WHO-surveillance-standard.pdf, accessed 19 January 2016).

7. Elsaid MF, Flamerzi AA, Bessisso MS, Elshafie SS. Acute bacterial meningitis in Qatar. Saudi Med J. 2006 Feb;27(2):198-204. PMID:16501676

8. Dash N, Al Khusaiby S, Behlim T, Mohammadi A, Mohammadi E, Al Awaidy S. Epidemiology of meningitis in Oman, 20002005. East Mediterr Health J. 2009 Nov-Dec;15(6):1358-64. PMID:20218125

9. Dash N, Ameen AS, Sheek-Hussein MM, Smego RA Jr. Epidemiology of meningitis in Al-Ain, United Arab Emirates, 20002005. Int J Infect Dis. 2007 Jul;11(4):309-12. PMID:16950640

10. Peltola H. Worldwide Haemophilus influenzae type b disease at the beginning of the 21st century: global analysis of the disease burden 25 years after the use of the polysaccharide vaccine and a decade after the advent of conjugates. Clin Microbiol Rev. 2000 Apr;13(2):302-17. PMID: 10756001

11. Epidemiology and prevention of vaccine preventable diseases, 13th ed. Atlanta (GA): Centers for Disease Control and Prevention; 2015 (http//www.cdc.gov/vaccines/puls/pinkbook, accessed 16 January 2016).

12. Shabani IS, Al-Ateeqi W, Abu-Shanab O, El-Sori H, Omar N, Ahmed HF, et al. Childhood meningitis in Kuwait: epidemiology of etiologic agents and the need for pneumococcal disease prevention. Med Princ Pract. 2006;15(6):431-5. PMID:17047350

13. Tsai C, R. Griffin M, Nuorti P, Grijalva C. Changing epidemiology of pneumococcal meningitis after the introduction of pneumococcal conjugate vaccine in the United States. Clin Infect Dis. 2008 Jun 1;46(11):1664-72. PMID:18433334
14. Dery MA, Hasbun R. Changing epidemiology of bacterial meningitis. Curr Infect Dis Rep. 2007 Jul;9(4):301-7. PMID:17618550

15. Thigpen MC, Whitney CG, Messonnier NE, Zell ER, Lynfield R, Hadler JL, et al.; Emerging Infections Programs Network. Bacterial meningitis in the United States, 1998-2007. N Engl J Med. 2011 May 26;364(21):2016-25. PMID:21612470

16. Martin NG, Sadarangani M, Pollard AJ, Goldacre MJ. Hospital admission rates for meningitis and septicaemia caused by Haemophilus influenzae, Neisseria meningitidis, and Streptococcus pneumoniae in children in England over five decades: a population-based observational study. Lancet Infect Dis. 2014 May;14(5):397-405. 10.1016/S1473-3099(14)70027-1 PMID:24631222

17. Hausdorff WP, Hajjeh R, Al-Mazrou A, et al. The epidemiology of pneumococcal, meningococcal, and Haemophilus disease in the Middle East and North Africa (MENA) region-current status and needs. Vaccine. 2007 Mar 1;25(11):1935-44. PMID:17241707

18. International Coordinating Group on Vaccine Provision for Epidemic Meningitis Control. Global alert and response [Internet]. Geneva: World Health Organization; 2010 (http://www.who. int/csr/disease/meningococcal/icg/en/index.html, accessed 16 January 2016).

19. MacNeil JR, Bennett N, Farley MM, Harrison LH, Lynfield $\mathrm{R}$, Nichols $\mathrm{M}$, et al. Epidemiology of Infant Meningococcal Disease in the United States, 2006-2012. Pediatrics. 2015 Feb;135(2):e305-11. PMID: 25583921

20. Castelblanco RL, Lee M, Hasbun R. Epidemiology of bacterial meningitis in the USA from 1997 to 2010: a population-based observational study. Lancet Infect Dis. 2014 Sep;14(9):813-9. PMID:25104307

21. von Gottberg A, de Gouveia L, Tempia S, Quan V, Meiring S, von Mollendorf C, et al.; GERMS-SA Investigators. Effects of vaccination on invasive pneumococcal disease in South Africa. N Engl J Med. 2014 Nov 13;371(20):1889-99. PMID:25386897

22. Pneumococcal vaccines WHO position paper-2012. Wkly Epidemiol Rec. 2012 Apr 6;87(14):129-44. PMID:24340399

23. Memish ZA, Shibl AM. Consensus building and recommendations based on the available epidemiology of meningococcal disease in Gulf Cooperation Council States. Travel Med Infect Dis. 2011 Mar;9(2):60-6. PMID:21345738

24. Ceyhan M, Anis S, Htun-Myint L, Pawinski R, Soriano-Gabarró $M$, Vyse A. Meningococcal disease in the Middle East and North Africa: an important public health consideration that requires further attention. Int J Infect Dis. 2012 Aug;16(8):e574-82. PMID:22647750

25. Howidi M, Muhsin H, Rajah J. The burden of pneumococcal disease in children less than 5 years of age in Abu Dhabi, United Arab Emirates. Ann Saudi Med. 2011 Jul-Aug;31(4):356-9. PMID:21808110 